\title{
Efficacy and Safety of a Chewable Methylphenidate Extended-Release Tablet in Children with Attention-Deficit/Hyperactivity Disorder
}

\author{
Sharon B. Wigal, PhD, ${ }^{1}$ Ann Childress, MD, Sally A. Berry, MD, PhD, ${ }^{3}$ Heidi Belden, PharmD, \\ Faith Walters, PharmD, ${ }^{4}$ Phillip Chappell, MD, MBA, Nancy Sherman, BA, \\ John Orazem, $\mathrm{PhD}^{4}$ and Donna Palumbo, $\mathrm{PhD}^{4}$
}

\begin{abstract}
Objective: This phase 3, laboratory classroom study assessed the efficacy and safety of methylphenidate hydrochloride extendedrelease chewable tablets (MPH ERCT) compared with placebo in children with attention-deficit/hyperactivity disorder (ADHD). Methods: Following a 6-week, open-label, dose-optimization period, children 6-12 years of age $(n=90)$ with ADHD were randomly assigned to double-blind MPH ERCT at the final optimized dose $(20-60 \mathrm{mg} /$ day $)$ or placebo. After 1 week of doubleblind treatment, efficacy was assessed predose and $0.75,2,4,8,10,12$, and 13 hours postdose in a laboratory classroom setting. The primary efficacy measure was the average of postdose Swanson, Kotkin, Agler, M-Flynn, and Pelham (SKAMP) Rating Scale-Combined scores, analyzed using a mixed-model, repeated-measures analysis. Secondary efficacy measures included Permanent Product Measure of Performance (PERMP) total number of problems attempted and total number of problems correct. Safety assessments included adverse event (AE) monitoring and the Columbia-Suicide Severity Rating Scale (C-SSRS). Results: MPH ERCT treatment statistically significantly reduced the average of all postdose SKAMP-Combined scores versus placebo (least-squares mean difference [95\% confidence interval], $-7.0[-10.9,-3.1] ; p<0.001$ ). Statistically significant treatment differences in SKAMP-Combined scores were observed at 2 hours postdose through 8 hours postdose ( $p$ values $<0.001)$. Statistically significant differences between MPH ERCT and placebo in PERMP total number of problems attempted and total number of problems correct were observed at 0.75 hours postdose through 8 hours postdose ( $p$-values $\leq 0.049)$. Common AEs in the open-label period $(\geq 5 \%)$ were decreased appetite, upper abdominal pain, mood swings, irritability, insomnia, upper respiratory tract infection (URTI), dysgeusia, and headache; URTI was the only AE reported by $>1$ subject receiving MPH ERCT in the double-blind period (placebo: URTI, contusion, wound, and initial insomnia). No suicidal ideation or behavior was reported on the C-SSRS at baseline or at any postbaseline assessment.

Conclusions: MPH ERCT 20-60 mg significantly improved ADHD symptoms compared with placebo at 2 hours postdose through at least 8 hours postdose. MPH ERCT was generally safe and well tolerated, with a safety profile consistent with other MPH ER formulations. ClinicalTrials.gov Identifier: NCT01654250. www.clinicaltrials.gov/ct2/show/NCT01654250.
\end{abstract}

Keywords: attention-deficit/hyperactivity disorder, methylphenidate, drug formulation, treatment efficacy, safety, children

\section{Introduction}

T he Diagnostic and STatistical Manual of Mental Disorders, Fifth Edition (DSM-5) (American Psychiatric Association 2013) characterizes attention-deficit/hyperactivity disorder (ADHD) as a persistent pattern of inattention and/or hyperactivity- impulsivity that interferes with function or development. ADHD symptoms can disrupt academic progress and social functioning during formative childhood and adolescence (American Psychiatric Association 2013; Feldman and Reiff 2014; Sharma and Couture 2014). Untreated ADHD is associated with multiple negative outcomes, including increased risk of depression and anxiety,

\footnotetext{
${ }^{1}$ AVIDA Inc., Newport Beach, California.

${ }^{2}$ Center for Psychiatry and Behavioral Medicine, Inc., Las Vegas, Nevada.

${ }^{3}$ Tris Pharma, Inc., Monmouth Junction, New Jersey.

${ }^{4}$ Pfizer Inc., New York, New York.

${ }^{5}$ Pfizer Inc., Groton, Connecticut.

Funding: This research was sponsored by NextWave Pharmaceuticals, a wholly owned subsidiary of Pfizer, Inc.
}

(c) Sharon B. Wigal et al. 2017; Published by Mary Ann Liebert, Inc. This is an Open Access article distributed under the terms of the Creative Commons Attribution License, which permits unrestricted use, distribution, and reproduction in any medium, provided the original work is properly cited. 
suicide, motor vehicle collisions, substance abuse, educational underachievement, financial problems, and unemployment (Jerome et al. 2006; Lee et al. 2011; Das et al. 2012). ADHD can impose a significant financial burden on families, health systems, and schools (Birnbaum et al. 2005; Matza et al. 2005). Practice guidelines recommend a combination of medication and behavior therapy for the treatment of ADHD in children and adolescents 618 years of age (Pliszka 2007; American Academy of Pediatrics 2011). Methylphenidate (MPH)- and amphetamine-based psychostimulants have the U.S. Food and Drug Administration (FDA) approval for the treatment of ADHD and are considered the standard of care (Pliszka 2007; American Academy of Pediatrics 2011).

ADHD is the most common neurobehavioral disorder in schoolaged children. Parental reports indicate that more than 1 in 10 school-aged children (11\%; 6.4 million children) in the United States have received a diagnosis of ADHD from a healthcare provider (Visser et al. 2014). Left untreated, ADHD can negatively affect academic performance, and social and family functioning (Barkley 2002; Klassen et al. 2004). An important barrier to adequate treatment of ADHD with pharmacologic agents is patient nonadherence to treatment regimens, and although adverse effects and lack of efficacy are most commonly reported as reasons for discontinuation, dislike of medication and difficulties swallowing medication are also cited as reasons for discontinuing treatment in children and adolescents (Gajria et al. 2014). A survey of 304 parents of 702 children or adolescents indicated that $\sim 34 \%$ of children and adolescents had refused to swallow pills, and $\sim 30 \%-$ $70 \%$ were unable to easily swallow a pill, with percentages increasing in younger children and for swallowing larger pills or capsules (Polaha et al. 2008). Indeed, in a study designed to assess pill swallowing ability, 67/124 (54\%) children, 6-11 years of age, who were willing to try to swallow a tablet could not (Meltzer et al. 2006).

Prescribing information for several ADHD medication capsule formulations states that capsules can be opened and the contents sprinkled on applesauce for individuals who cannot swallow the capsule (Focalin XR package insert 2015; Metadate CD package insert 2015; Ritalin LA package insert 2015; Aptensio package insert 2017). However, there may be a risk of either over- or underdosing using this method (Childress and Sallee 2013). The accuracy of dosing in patients taking ADHD medication sprinkled on applesauce has not been examined systematically, but patient may be underdosed if some of the contents of the capsule are spilled while preparing the applesauce for dosing or if the patient does not ingest the entire amount of applesauce prepared. Although pharmacokinetic studies have demonstrated bioequivalence of ADHD capsule formulations swallowed versus sprinkled over applesauce (Pentikis et al. 2002; Lee et al. 2003; Fischer et al. 2006; Adjei et al. 2014), over- or underdosing is theoretically possible. Dose dumping (overdosing) could occur if the patient inadvertently bites down on long-acting beads in the applesauce, releasing the medication prematurely (Childress and Sallee 2013). The development of additional dosing and formulation options that take into account the abilities and preferences of patients ranging from children to adults may improve the acceptability of ADHD medications and improve adherence.

MPH was initially formulated as an immediate-release (IR) tablet requiring multiple daily doses for optimal treatment. Extendedrelease (ER) MPH formulations were later developed to provide all-day coverage, with the goal of eliminating the need for inschool administration, and to potentially increase efficacy and reduce adverse events (AEs) (Sugrue et al. 2014). Several long-acting MPH formulations are currently available, including oral capsules and tablets (Concerta package insert 2015; Metadate CD package insert 2015; Ritalin LA package insert 2015), an oral suspension (Quillivant XR package insert 2016), and the transdermal patch (Daytrana package insert 2015). The addition of a chewable tablet form of MPH ER to currently available MPH formulations would expand formulation options by providing a safe and well-tolerated, palatable, stable, and convenient formulation (Michele et al. 2002). ER chewable tablets offer a child-friendly alternative for patients or parents who are not satisfied with the available formulation options, especially those individuals who cannot or will not swallow tablets or capsules, and could improve treatment compliance.

MPH ER chewable tablets (MPH ERCT) are a once-daily chewable tablet formulation of MPH developed using proprietary ER technology. A pharmacokinetic study has demonstrated that the bioavailability of a $40 \mathrm{mg}$ MPH ERCT is comparable to that of $40 \mathrm{mg}$ IR MPH chewable tablet, given as two $20 \mathrm{mg}$ doses 6 hours apart in healthy adult subjects (Abbas et al. 2016). The current study was a dose-optimized, randomized, double-blind, placebocontrolled, laboratory classroom study of MPH ERCT for the treatment of children 6-12 years of age with ADHD. The study objectives were to assess the efficacy, safety, and tolerability of oncedaily MPH ERCT 20-60 mg in pediatric subjects with ADHD.

\section{Methods}

This phase 3, laboratory classroom study was carried out at six U.S. sites between July 2012 and October 2012. The study was conducted in accordance with the International Harmonization Guideline for Good Clinical Practice (International Council for Harmonisation 1998) and other applicable regulatory requirements. Parents or guardians provided written informed consent and children gave assent before any protocol-required procedures were performed. The protocol, consent and assent forms, and the investigator's brochure received institutional review board approval before initiation of the study.

\section{Study subjects}

Eligible subjects were males and females 6-12 years of age with a diagnosis of ADHD determined by a psychiatrist, developmental pediatrician, pediatrician, or licensed allied health professional, who were deemed to have the need for pharmacological treatment for ADHD in the judgment of the investigator. Diagnoses were confirmed based on three criteria: positive confirmation of ADHD diagnosis using the Schedule for Affective Disorders and Schizophrenia for School Age Children (K-SADS) (Kaufman et al. 1997) semistructured diagnostic interview at screening by a psychiatrist, psychologist, developmental pediatrician, pediatrician, or licensed clinical social worker, trained and experienced with the K-SADS (visit 1); investigator administered Clinical Global ImpressionsSeverity (CGI-S) (Guy 1976) score of at least 3 (mildly ill); and an investigator administered ADHD Rating Scale (ADHD-RS-IV) Home Version score in the 90th percentile or greater for gender and age on the hyperactive-impulsive subscale, inattentive subscale, and/or total score at screening or baseline (visit 2).

Subjects were excluded from the study if they had a current primary psychiatric diagnosis of severe anxiety disorder, conduct disorder, pervasive developmental disorder, eating disorder, obsessivecompulsive disorder, major depressive disorder, bipolar disorder, or other psychiatric disorder, substance abuse disorder, or a personal or family history of Tourette's syndrome. Subjects were also excluded if they had a clinically significant or severe medical illness or condition, including seizure disorder, cardiac disorders or 
conditions (including severe hypertension), untreated thyroid disease, or a history of HIV or hepatitis B or C infections. Subjects with clinically significant abnormal laboratory results or a positive test for illicit drug use at screening were excluded, as were those with a history of hypersensitivity or lack of efficacy to MPH. Psychotropic agents were prohibited. Sedative hypnotics were prohibited within 24 hours before screening, except sedative hypnotics that had been prescribed as sleep aids (at bedtime only) for at least 30 days before the baseline visit. (Promethazine [one MPH ERCTtreated patient, for viral infection] was the only sedative hypnotic reported in this study.) Pharmacologic treatments for ADHD, including noninvestigational stimulant medications for the control of ADHD, were allowed until 24 hours before the baseline visit for the open-label, dose-optimization period.

\section{Study design}

The study included a 6-week, open-label, dose-optimization treatment period followed by a 1-week, randomized, double-blind, placebo-controlled period. During the open-label period, subjects received MPH ERCT at a starting dose of $20 \mathrm{mg}$ once daily. At weekly intervals during the open-label period (visits 3-8), the dose could be increased, in $10-20 \mathrm{mg} /$ day increments to a maximum dose of $60 \mathrm{mg} /$ day, or decreased to optimize efficacy and tolerability. Dose adjustments were based on clinical judgment of the investigator taking into account all available efficacy and tolerability information. Subjects who could not tolerate $20 \mathrm{mg} /$ day or were unable to achieve a stable dose during the open-label period were discontinued. At the final open-label visit (visit 8), subjects were assessed in an abbreviated, 4-hour practice laboratory classroom visit (McGough et al. 2006; Wigal et al. 2009, 2014; Childress et al. 2015) designed to acclimate the subjects to the classroom setting.

The randomized, double-blind treatment period included 1 week of double-blind treatment followed by a laboratory classroom evaluation (visit 9). Subjects were randomly assigned (1:1) to receive either MPH ERCT, at their optimal open-label dose (20-60 mg/ day), or placebo daily for 1 week. Randomization was conducted according to a fixed schedule using a permuted block design stratified by clinical site. The randomization code was maintained centrally by the clinical supply group, and the study team and investigator site personnel were blinded throughout the study. On the final day of the double-blind treatment period, efficacy assessments were administered in a laboratory classroom day using the laboratory school protocol, a tool for evaluating response to ADHD treatments in a setting that reflects a real-world school day environment and activities (Wigal and Wigal 2006). The laboratory school protocol is designed to approximate school day and afterschool activities while incorporating efficacy and safety assessments (Wigal et al. 2013). A follow-up (visit 10) was scheduled 1-2 weeks after the double-blind visit, or at early termination.

\section{Assessments}

Efficacy. Efficacy of open-label treatment was assessed for dose optimization using the ADHD-RS-IV, Conners Parent Rating Scale (Conners et al. 1998), CGI-S, and Clinical Global ImpressionsImprovement (CGI-I) (Guy 1976) administered at baseline (except CGI-I) and at weekly visits during the open-label period; these assessments were not administered at the double-blind visit. Subjects were assessed by trained raters using the Swanson, Kotkin, Agler, M-Flynn, and Pelham (SKAMP) Rating Scale (Wigal et al. 1998) and completed the Permanent Product Measure of Perfor- mance (PERMP) math test (Wigal and Wigal 2006) during the practice laboratory classroom visit.

After the 1-week, double-blind, treatment period, ADHD symptoms were evaluated during the laboratory classroom session using the SKAMP and PERMP assessments administered predose and at $0.75,2,4,8,10,12$, and 13 hours postdose. The primary efficacy endpoint for the study was the average of all the postdose SKAMPCombined scores assessed during visit 9, the classroom study day. The SKAMP scale is a validated, 13-item rating of the level of impairment of classroom-observed behaviors, with each item rated on a seven-point scale from 0 (none) to 6 (maximal impairment) (Wigal and Wigal 2006). The SKAMP-Combined score is calculated as the total of all 13 items, with a higher score indicating greater impairment. Key secondary endpoints were the onset and duration of the clinical efficacy of MPH ERCT compared with placebo based on the SKAMP-Combined scores obtained at each time point during the laboratory classroom session.

Additional efficacy endpoints based on the SKAMP assessment were the SKAMP-Attention subscale score (total of items 1-4) and SKAMP-Deportment subscale score (total of items 5-8) at visit 9, and PERMP scores at visit 9. The PERMP, a timed, skill-adjusted, mathematics test designed to measure a child's ability to attend to written seatwork, was scored on the total number of problems attempted and the total number of problems correct. Subjects were asked to complete as many of the 400 total problems as they could during a 10-minute period. Each subject's skill level was determined using a PERMP pretest at screening, and a different version of the test, matched to the subject's pretest skill level, was administered at each study assessment.

Safety. Safety assessments included the incidence of AEs and serious AEs, clinical laboratory tests, measurements of vital signs, physical examinations, 12-lead electrocardiograms (ECG), and the Columbia-Suicide Severity Rating Scale (C-SSRS) (Posner et al. 2011), a prospective and systematic measurement of suicidal thoughts and behaviors designed for use in clinical trials (Posner et al. 2007). The screening evaluation included physical examination, vital sign and ECG measurements, and laboratory evaluations. Safety and tolerability were assessed weekly during the open-label period and at the double-blind study visit using the C-SSRS, vital sign measurements, and AE queries. The follow-up visit included physical examination, vital sign and ECG measurements, laboratory evaluations, and assessment of AEs. The investigators who recorded the AEs did not conduct the efficacy evaluations, and the classroom raters collecting efficacy data had no duties outside the classroom and were not aware of AEs collected by the investigators.

\section{Statistical analysis}

Efficacy analyses were based on the intent-to-treat (ITT) population, which included all subjects who received at least one dose of the study drug and had at least one postbaseline assessment of the primary efficacy variable. Safety assessments were based on the enrolled safety population (all enrolled subjects who received at least one dose of study medication and had at least one postbaseline safety assessment), including safety data from the open-label, doseoptimization period, and the randomized safety population (all randomized subjects who received at least one dose of double-blind study medication and had at least one postbaseline safety assessment), including safety data from the double-blind period only.

The primary efficacy analysis was a mixed-model, repeatedmeasures (MMRM) analysis with intercept as the random effect 
and treatment, study center, time point, and the time point by treatment interaction as fixed effects. The average difference over all postdose time points was estimated using least-squares (LS) means from the MMRM model. The comparison between treatment groups was a two-sided test at the 0.05 level of significance. To test treatment differences in the SKAMP-Combined score at each postdose time point, a fixed-sequence testing procedure was applied to adjust for multiple comparisons to placebo in accordance with the study protocol. The fixed-sequence procedure assessed treatment differences in the following prespecified order: $4,8,2$, $10,12,13$, and 0.75 hours; the first time a treatment difference had $p>0.05$ caused the remaining treatment differences to be considered not statistically significant, regardless of the nominal $p$-value. The order for the fixed-sequence testing procedure was selected $a$ priori to test time points starting with the most likely to be significant and moving to the least (based on the time course of other MPH ER formulations) (Pelham et al. 2001; McGough et al. 2006; Kimko et al. 2012; Wigal et al. 2014). Additional secondary efficacy variables, including SKAMP-Attention, SKAMP-Deportment, PERMP-total number of problems attempted, and PERMP-total number of problems correct, also were analyzed by time point using the same MMRM analysis. Because SKAMP subscales (attention, deportment) and PERMP variables were not declared as primary or key secondary endpoints, a multiple comparisons adjustment was not applied in these analyses according to the study protocol.

To adjust for numerical imbalance between treatment groups in predose scores, post hoc efficacy analyses of change from predose in SKAMP-Combined, SKAMP-Attention, SKAMP-Deportment, and PERMP scores at each time point at the double-blind study visit were conducted, using the same MMRM model used in the primary efficacy analysis. Because of the post hoc nature of these analyses, the Bonferroni method was used to adjust for multiple comparisons for all changes from predose analyses.

Demographic characteristics and safety data, including C-SSRS scores, and incidence of AEs and serious AEs, were summarized descriptively by treatment group.

\section{Results}

\section{Subject disposition}

Ninety subjects were enrolled in the study (Supplementary Fig. S1; Supplementary Data are available online at www.liebertpub.com/ cap). Four subjects discontinued during the open-label period (3 withdrew consent; 1 discontinued due to $\mathrm{AE}$ [dysgeusia, bad taste of medicine]), and 86 were randomly assigned to treatment (placebo, $n=44$; MPH ERCT, $n=42$ ). A total of 85 subjects received at least one dose of double-blind study medication and had at least one postbaseline efficacy evaluation and were included in the randomized safety population and ITT population. One subject assigned to placebo discontinued the study during the double-blind treatment period (lost to follow-up). Baseline demographics and clinical characteristics of subjects included in the ITT population $(n=85)$ are summarized in Table 1. The mean (standard deviation [SD]) age of subjects was 9.6 (1.69) years; the majority was white $(58 \% ; 35 \%$ black, $85 \%$ non-Hispanic/Latino) and male (62\%). Subjects had inattentive $(27 \%)$ or combined type (73\%) ADHD. The majority of subjects $(81 \%)$ had no exposure to MPH or dexmethylphenidate hydrochloride within 1 month before screening; 7/42 (17\%) subjects in the MPH ERCT group and 9/44 (20\%) subjects in the placebo group had prior (within 1 month) exposure to MPH or dexmethylphenidate hydrochloride.
Table 1. Baseline Demographics and Clinical Characteristics, Intent-to-Treat Population

\begin{tabular}{|c|c|c|c|}
\hline & $\begin{array}{l}\text { Placebo } \\
(\mathrm{n}=43)\end{array}$ & $\begin{array}{c}M P H E R C T \\
(\mathrm{n}=42)\end{array}$ & $\begin{array}{c}\text { Total } \\
(\mathrm{n}=85)\end{array}$ \\
\hline \multicolumn{4}{|l|}{ Gender, $n(\%)$} \\
\hline Male & $23(53.5)$ & $30(71.4)$ & $53(62.4)$ \\
\hline Female & $20(46.5)$ & $12(28.6)$ & $32(37.6)$ \\
\hline Mean $\pm S D$ age, years & $9.3(1.62)$ & $9.9(1.71)$ & $9.6(1.69)$ \\
\hline \multicolumn{4}{|l|}{ Age categories, years $(\%)$} \\
\hline $6-7$ & $8(18.6)$ & $5(11.9)$ & $13(15.3)$ \\
\hline $8-10$ & $28(65.1)$ & $17(40.5)$ & $45(52.9)$ \\
\hline $11-12$ & $7(16.3)$ & $20(47.6)$ & $27(31.8)$ \\
\hline \multicolumn{4}{|l|}{ Race, $n(\%)$} \\
\hline White & $22(51.2)$ & $27(64.3)$ & $49(57.6)$ \\
\hline $\begin{array}{c}\text { Black/African } \\
\text { American }\end{array}$ & $18(41.9)$ & $12(28.6)$ & $30(35.3)$ \\
\hline Asian & $1(2.3)$ & 0 & $1(1.2)$ \\
\hline Other & $2(4.7)$ & $3(7.1)$ & $5(5.9)$ \\
\hline \multicolumn{4}{|l|}{ Ethnicity, $n(\%)$} \\
\hline Hispanic/Latino & $6(14.0)$ & $7(16.7)$ & $13(15.3)$ \\
\hline Non-Hispanic/Latino & $37(86.0)$ & $35(83.3)$ & $72(84.7)$ \\
\hline \multicolumn{4}{|l|}{ ADHD type, $n(\%)$} \\
\hline Inattentive & $11(25.6)$ & $12(28.6)$ & $23(27.1)$ \\
\hline Hyperactive/impulsive & 0 & 0 & 0 \\
\hline Combined & $32(74.4)$ & $30(71.4)$ & $62(72.9)$ \\
\hline
\end{tabular}

ADHD, attention-deficit/hyperactivity disorder; ADHD-RS, AttentionDeficit/Hyperactivity Disorder Rating Scale; MPH ERCT, methylphenidate hydrochloride extended-release chewable tablets; SD, standard deviation.

\section{Efficacy}

Treatment with MPH ERCT was associated with a statistically significant reduction in ADHD symptoms compared with placebo based on the primary efficacy endpoint, average of all postdose SKAMP-Combined scores at visit 9 (Fig. 1). Averaged overall postdose time points, SKAMP-Combined scores were significantly lower for patients treated with MPH ERCT compared with those treated with placebo (LS mean difference [95\% confidence interval], -7.0 [-10.9, -3.1]; $p<0.001$; effect size [Cohen's $d$ ], 0.66). The primary endpoint remained significant after adjustment for additional prognostic factors (age, gender, dose, weight, race, and ADHD subtype).

Onset and duration of efficacy were assessed based on the key secondary efficacy endpoints, SKAMP-Combined scores at each time point at visit 9 (Fig. 2). There were statistically significant differences in SKAMP-Combined scores between MPH ERCT and placebo from 2 hours postdose and continuing through 8 hours postdose after adjusting for the prespecified fixed-sequence testing procedure $(p<0.001$ at 2,4 , and 8 hours postdose; Table 2$)$. The 10-hour comparison did not reach statistical significance ( $p=0.133)$, and all subsequent comparisons in the fixed sequence (12-, 13-, and 0.75-hour time points) were considered nonsignificant. Effect sizes were $0.72,1.16,1.13,0.66,0.26,0.19$, and 0.08 at $0.75,2,4,8,10,12$, and 13 hours postdose, respectively. Nominal $p$-values are given in Table 2. Predose SKAMP-Combined scores were numerically greater for the MPH ERCT treatment group versus the placebo group (Table 2), although the difference did not reach statistical significance $(p>0.05)$.

Results for other secondary efficacy endpoints, SKAMPsubscale scores and PERMP total number of problems attempted and total number of problems correct, were consistent with the 


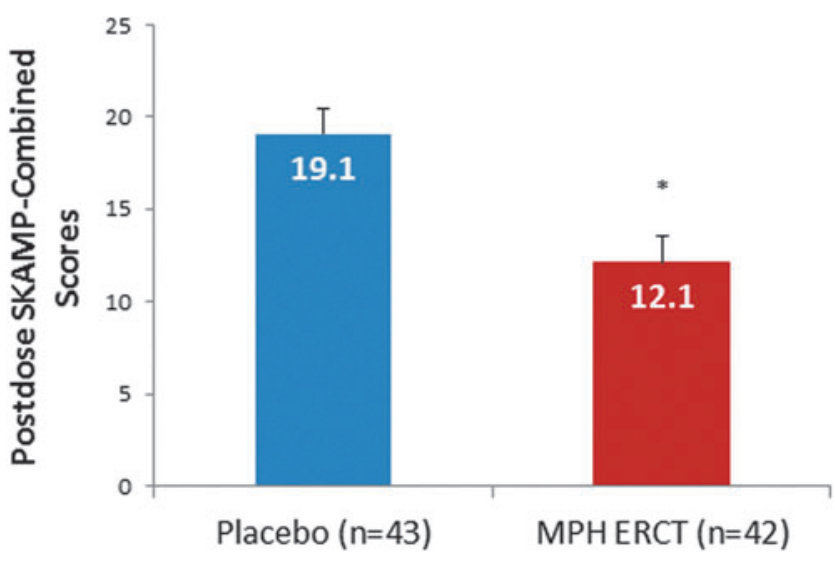

FIG. 1. SKAMP-Combined scores at visit 9, average over all postdose time points; intent-to-treat population. ${ }^{*} p<0.001$. SKAMP, Swanson, Kotkin, Agler, M-Flynn, and Pelham.

primary and key secondary results (Supplementary Fig. S2). Both the SKAMP-Attention and SKAMP-Deportment scores were significantly lower for MPH ERCT compared with placebo at $0.75,2$, 4, and 8 hours postdose (Table 3). Subjects in the MPH ERCT treatment group attempted a significantly higher number of problems on the PERMP compared with subjects in the placebo group and answered a significantly higher number of problems correctly at the 0.75-, 2-, 4-, and 8-hour time points (Supplementary Table S1). For the average overall postdose time points, the PERMP total number of problems attempted was significantly greater for subjects treated with MPH ERCT compared with subjects treated with placebo (LS mean difference [95\% confidence interval], 24.5 [4.4, 44.7]; $p=0.017)$; the treatment difference for total number correct approached significance $(20.5[-0.3,41.4] ; p=0.054)$.

Change from predose score at each postdose time point was explored for each measure in a post hoc analysis to adjust for the numerical imbalance between treatment groups observed at the predose time point. In the analysis of change from predose SKAMP-Combined scores, Bonferroni-adjusted statistically significant differences between MPH ERCT and placebo groups were observed at 0.75 hours postdose, and continuing through 12 hours postdose (all $p \leq 0.007$; Table 4 ). In the analyses of change from predose, Bonferroni-adjusted statistically significant differences between MPH ERCT and placebo groups were observed at 0.75, 2, 4 , and 8 hours postdose for both SKAMP-Attention and SKAMPDeportment scores (all $p \leq 0.007$; Supplementary Table S2). Bonferroni-adjusted statistically significant differences in change from predose in PERMP total number of problems attempted and PERMP problems correct were observed at all postdose time points (0.75-13 hours postdose; all $p \leq 0.007$; Supplementary Table S3).

\section{Safety}

The mean (SD) daily dose of MPH ERCT for the entire study was $33.0 \mathrm{mg}$. Double-blind MPH ERCT dose assignments, based on final, open-label, daily dose, were $20 \mathrm{mg} /$ day, $n=4 ; 30 \mathrm{mg} /$ day, $n=4 ; 40 \mathrm{mg} /$ day, $n=15 ; 50 \mathrm{mg} /$ day, $n=9$; and $60 \mathrm{mg} /$ day, $n=10$. The mean final dose for the enrolled safety population was $42.5 \mathrm{mg}$, and for the randomized safety population was $42.8 \mathrm{mg}$.

Treatment-emergent AEs (TEAEs) were reported by 65/90 (72\%) subjects in the open-label period. The most commonly reported TEAEs in the open-label period are summarized in Table 5. A total of 24 subjects reported TEAEs during the double-blind treatment period with a similar frequency between treatment groups (placebo, 13/44 [29.5\%]; MPH ERCT, 11/42 [26.2\%]). The only TEAE reported by more than one subject receiving MPH ERCT in the double-blind period was upper respiratory tract infection (URTI), reported by $3(7 \%)$ subjects in each treatment group. No severe AEs or serious AEs were reported, and no deaths occurred at any time during the study. No subjects reported suicidal ideation or behavior on the C-SSRS at baseline or at any postbaseline assessment. One subject reported nonsuicidal selfinjurious behavior at open-label week 4 . The subject abraded the skin of the inner forearm with an eraser, mimicking the behavior of

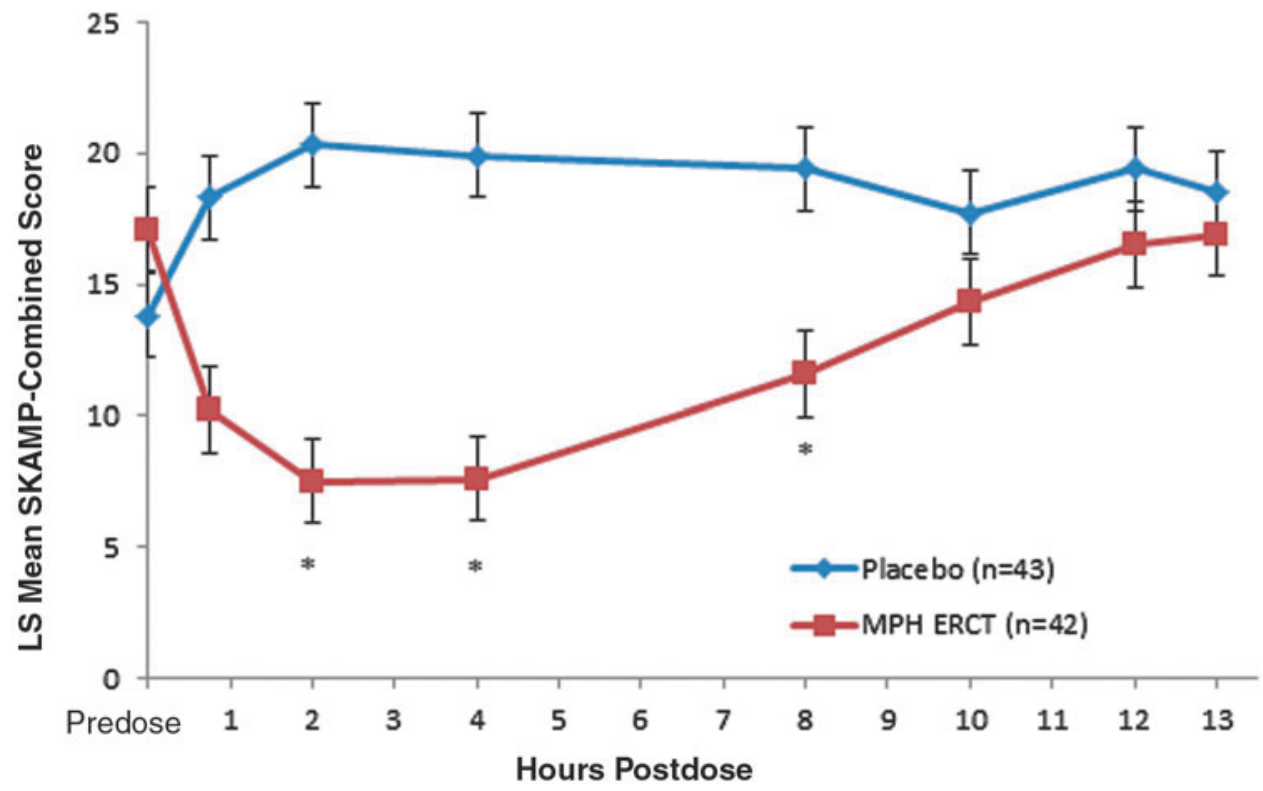

FIG. 2. Least squares mean (standard error) SKAMP-Combined scores at visit 9 , intent-to-treat population. $* p<0.001$. 
Table 2. SKaMP-Combined Scores at Visit 9, Intent-to-Treat Population

\begin{tabular}{|c|c|c|c|c|c|}
\hline & Placebo & $M P H E R C T$ & $\begin{array}{l}\text { Treatment difference } \\
\text { (MPH ERCT-placebo) }\end{array}$ & $\begin{array}{l}\text { Nominal } \\
\text { p-value }\end{array}$ & $\begin{array}{l}\text { Adjusted } \\
\text { p-value }\end{array}$ \\
\hline Predose, mean (SD) & $13.8(10.03)$ & $17.5(11.56)$ & & & \\
\hline \multicolumn{6}{|c|}{ Postdose, LS mean (SE), hours } \\
\hline 0.75 & $18.3(1.60)$ & $10.2(1.62)$ & $-8.2(2.28)$ & $<0.001$ & NS* \\
\hline 2 & $20.3(1.60)$ & $7.5(1.62)$ & $-12.8(2.28)$ & $<0.001$ & $<0.001 *$ \\
\hline 4 & $19.9(1.60)$ & $7.6(1.62)$ & $-12.3(2.28)$ & $<0.001$ & $<0.001 *$ \\
\hline 8 & $19.4(1.60)$ & $11.6(1.62)$ & $-7.8(2.28)$ & $<0.001$ & $<0.001 *$ \\
\hline 10 & $17.7(1.60)$ & $14.3(1.62)$ & $-3.4(2.28)$ & 0.133 & NS* \\
\hline 12 & $19.4(1.60)$ & $16.5(1.62)$ & $-2.9(2.28)$ & 0.206 & NS* \\
\hline 13 & $18.5(1.60)$ & $16.9(1.62)$ & $-1.6(2.28)$ & 0.496 & NS* \\
\hline
\end{tabular}

* $p$-values adjusting for the fixed-sequence testing procedure.

LS, least squares; MPH ERCT, methylphenidate hydrochloride extended-release chewable tablets; NS, not significant; SD, standard deviation; SE, standard error; SKAMP, Swanson, Kotkin, Agler, M-Flynn, and Pelham Rating Scale.

classmates. The subject was interviewed and was found to have no suicidal thoughts. The subject had no suicidal thoughts or behaviors before or following this event and completed the study.

One subject in the placebo group had two clinically significant changes in laboratory results (elevated prothrombin time and partial thromboplastin time at visit 1). The findings resolved with no subsequent elevations and no sequelae. After medical review, the subject was allowed to continue in the study. A small mean [SD] increase from baseline in blood pressure was observed during the open-label period (diastolic, 2.0 [7.76] $\mathrm{mmHg}$; systolic, 1.9 [8.97] $\mathrm{mmHg}$ ). At the double-blind visit, mean [SD] change in blood pressure from baseline was similar for placebo (diastolic, 2.4 [7.22] $\mathrm{mmHg}$; systolic, 3.0 [8.52] $\mathrm{mmHg}$ ) and MPH ERCT groups (diastolic, 1.3 [7.05] mmHg; systolic, 1.5 [8.95] mmHg). Potentially clinically significant (PCS) vital sign values observed during the entire study included postbaseline blood pressure values greater than the 95th percentile (systolic, 17/90 subjects, diastolic, 8/90 subjects), PCS increase from baseline blood pressure (systolic increase $\geq 20 \mathrm{mmHg}, 7 / 90$; diastolic increase $\geq 10 \mathrm{mmHg}, 37 / 90$ ), postbaseline pulse rate greater than $110 \mathrm{bpm}(8 / 90)$, and PCS increase from baseline pulse rate (pulse rate increase $\geq 25 \mathrm{bpm}, 17 /$ 90). A total of 13 subjects had PCS diastolic blood pressure readings (placebo, 7; MPH ERCT, 6) and 4 subjects had PCS systolic blood pressure readings (placebo, 2; MPH ERCT, 2) during the double-blind period. Three subjects receiving double-blind MPH ERCT had PCS increased pulse values (placebo, 0). PCS vital sign changes were generally transient. Two PCS systolic blood pressure values, 15 PCS diastolic blood pressure values, and 1 PCS pulse rate occurred at last visit; however, only 11 subjects with PCS vital sign findings during the study had a follow-up visit. In those subjects, 2 PCS systolic blood pressure values, 4 PCS diastolic blood pressure values, and 0 PCS pulse rate findings were observed at follow-up. No abnormal physical examination findings were observed at follow-up. Two subjects assigned to placebo had

Table 3. SKaMP Subscale Scores at Visit 9, Intent-to-Treat Population

\begin{tabular}{|c|c|c|c|c|}
\hline & Placebo & $M P H E R C T$ & $\begin{array}{l}\text { Treatment difference } \\
\text { (MPH ERCT-placebo) }\end{array}$ & $\mathrm{p}$-Value \\
\hline \multicolumn{5}{|l|}{ SKAMP-Attention } \\
\hline Predose, mean (SD) & $2.7(2.78)$ & $3.3(3.71)$ & & \\
\hline \multicolumn{5}{|c|}{ Postdose, LS mean (SE), hours } \\
\hline 0.75 & $2.9(0.40)$ & $1.4(0.40)$ & $-1.5(0.56)$ & 0.007 \\
\hline 2 & $3.5(0.40)$ & $1.0(0.40)$ & $-2.5(0.56)$ & $<0.001$ \\
\hline 4 & $3.2(0.40)$ & $0.9(0.40)$ & $-2.3(0.56)$ & $<0.001$ \\
\hline 8 & $3.5(0.40)$ & $1.9(0.40)$ & $-1.7(0.56)$ & 0.003 \\
\hline 10 & $3.4(0.40)$ & $2.5(0.40)$ & $-0.9(0.56)$ & 0.097 \\
\hline 12 & $3.4(0.40)$ & $3.0(0.40)$ & $-0.4(0.56)$ & 0.490 \\
\hline 13 & $3.8(0.40)$ & $3.0(0.40)$ & $-0.8(0.56)$ & 0.164 \\
\hline \multicolumn{5}{|l|}{ SKAMP-Deportment } \\
\hline Predose, mean (SD) & $3.0(3.83)$ & $3.1(3.80)$ & & \\
\hline \multicolumn{5}{|c|}{ Postdose, LS mean (SE), hours } \\
\hline 0.75 & $4.6(0.66)$ & $1.9(0.67)$ & $-2.7(0.94)$ & 0.004 \\
\hline 2 & $5.4(0.66)$ & $1.4(0.67)$ & $-3.9(0.94)$ & $<0.001$ \\
\hline 4 & $5.4(0.66)$ & $1.5(0.67)$ & $-3.9(0.94)$ & $<0.001$ \\
\hline 8 & $4.3(0.66)$ & $2.4(0.67)$ & $-1.9(0.94)$ & 0.042 \\
\hline 10 & $3.9(0.66)$ & $2.4(0.67)$ & $-1.5(0.94)$ & 0.118 \\
\hline 12 & $4.2(0.66)$ & $3.3(0.67)$ & $-0.9(0.94)$ & 0.342 \\
\hline 13 & $3.7(0.66)$ & $3.7(0.67)$ & $0(0.94)$ & 0.962 \\
\hline
\end{tabular}

LS, least squares; MPH ERCT, methylphenidate hydrochloride extended-release chewable tablets; SD, standard deviation; SE, standard error; SKAMP, Swanson, Kotkin, Agler, M-Flynn, and Pelham Rating Scale. 
Table 4. Least Squares Mean (Standard Error) Change from Predose For SKAMP-Combined Scores at Visit 9, Post Hoc Analysis

\begin{tabular}{|c|c|c|c|c|c|}
\hline & Placebo & $M P H E R C T$ & $\begin{array}{l}\text { Treatment difference } \\
\text { (MPH ERCT-placebo) }\end{array}$ & $\mathrm{p}$-Value & $\begin{array}{c}\text { Significant with } \\
\text { Bonferroni adjustment* }\end{array}$ \\
\hline 0.75 hours & $4.5(1.30)$ & $-6.9(1.30)$ & $-11.5(1.8)$ & $<0.001$ & $\mathrm{Y}$ \\
\hline 2 hours & $6.5(1.30)$ & $-9.6(1.30)$ & $-16.1(1.8)$ & $<0.001$ & $\mathrm{Y}$ \\
\hline 4 hours & $6.1(1.30)$ & $-9.5(1.30)$ & $-15.6(1.8)$ & $<0.001$ & Y \\
\hline 8 hours & $5.6(1.30)$ & $-5.5(1.30)$ & $-11.1(1.8)$ & $<0.001$ & $\mathrm{Y}$ \\
\hline 10 hours & $3.9(1.30)$ & $-2.8(1.30)$ & $-6.7(1.8)$ & $<0.001$ & $\mathrm{Y}$ \\
\hline 12 hours & $5.6(1.30)$ & $-0.6(1.30)$ & $-6.2(1.8)$ & $<0.001$ & $\mathrm{Y}$ \\
\hline 13 hours & $4.7(1.30)$ & $-0.2(1.30)$ & $-4.9(1.8)$ & 0.0076 & $\mathrm{~N}$ \\
\hline
\end{tabular}

*Statistical significance after Bonferroni correction: $p \leq 0.007$.

LS, least squares; MPH ERCT, methylphenidate hydrochloride extended-release chewable tablets; SKAMP, Swanson, Kotkin, Agler, M-Flynn, and Pelham Rating Scale.

abnormal ECG results at screening (QRS prolongation and right bundle branch block). Neither finding was considered clinically significant; both subjects completed the study.

\section{Discussion}

In this first multicenter, phase 3 study of the efficacy and safety of an MPH ERCT, statistically significant improvement in behavior impairment was demonstrated in a laboratory school setting in children with ADHD. The study's primary efficacy endpoint, the average of all postdose SKAMP-Combined scores, was statistically significantly reduced with MPH ERCT $20-60 \mathrm{mg}$ /day treatment compared with placebo. The SKAMP-Combined score findings were supported by the results from both SKAMP subscales and the PERMP number of problems attempted and number of problems correct. Evidence of MPH ERCT efficacy using the laboratory classroom study design is clinically important, demonstrating that improvements in impairment are observed during activities and in a setting that approximate those of a typical elementary school day (Wigal and Wigal 2006).

Onset and duration of efficacy were assessed using SKAMPCombined scores at each postdose time point (key secondary efficacy endpoints) and change from predose SKAMP-Combined scores at each postdose time point. The analysis of SKAMP-Combined scores at postdose time points showed a significant treatment effect at 2 hours postdose, with efficacy maintained through at least 8 hours postdose. Significant improvements on SKAMP-Attention and

Table 5. Treatment-Emergent Adverse Events Occurring With an INCIDENCE $\geq 5 \%$ In The Open-Label, Dose Optimization Period; Enrolled Safety Population, $N(\%)$

\section{TEAE}

Any TEAE

Decreased appetite

Upper abdominal pain

Mood swings

Irritability

Insomnia

Upper respiratory tract infection

Dysgeusia

Headache

MPH ERCT $(\mathrm{n}=90)$

$65(72.2)$

$33(36.7)$

$13(14.4)$

$12(13.3)$

$12(13.3)$

$10(11.1)$

$10(11.1)$

$8(8.9)$

$8(8.9)$
MPH ERCT, methylphenidate hydrochloride extended-release chewable tablets; TEAE, treatment-emergent adverse event.
Deportment subscale scores and PERMP total number of problems attempted and total number of problems correct were observed from 0.75 to 8 hours postdose in the prespecified analyses. Although the nominal $p$-value at the 0.75 -hour postdose time point was $<0.001$, the 0.75 -hour comparison was declared nonsignificant because a nonsignificant endpoint (10 hours postdose) preceded it in the fixed-sequence testing order. Therefore, the treatment effect at the 0.75 -hour postdose time point was not statistically significant after adjusting for the fixed-sequence testing procedure. However, there was a numerical imbalance between treatment groups in mean SKAMP-Combined scores at the predose time point (13.8 for placebo vs. 17.5 for MPH ERCT). To adjust for this imbalance, a post hoc analysis of change from predose SKAMP-Combined scores was conducted, and the results of that analysis showed a significant effect of MPH ERCT versus placebo on change from predose value at time points from 0.75 through 12 hours postdose. The change from predose measure is commonly reported in laboratory classroom studies in the literature (Childress et al. 2015; Wigal et al. 2009), and the inclusion of the post hoc analysis allows comparisons with findings from previous studies of other ADHD treatments in children. The results of the SKAMP-Combined post hoc analysis suggest that MPH ERCT has an onset/duration profile consistent with other MPH formulations (Swanson et al. 2004; McGough et al. 2006; Wigal et al. 2013, 2014). Given the differences between results of the prespecified and post hoc analyses of SKAMP-Combined scores at each time point, however, additional studies are needed to confirm that MPH ERCT and other MPH preparations have the same onset and duration.

Treatment with MPH ERCT was safe and generally well tolerated in children 6-12 years of age, with a safety and tolerability profile similar to that of other MPH ER formulations (Wolraich et al. 2007; Robb et al. 2014; Wigal et al. 2014). In the open-label, dose-optimization period, the most commonly reported TEAEs were consistent with those observed in children taking other MPH ER formulations for ADHD: decreased appetite, headache, abdominal pain, mood swings, irritability, and insomnia were among the most common AEs reported during the open-label, doseoptimization period of similarly designed studies of MPH ER capsule (Wigal et al. 2014) and MPH ER oral suspension formulations (Robb et al. 2014). AEs associated with MPH treatment generally occur early in treatment (Wolraich et al. 2007; Sugrue et al. 2014), and in the current study, fewer TEAEs were reported during the double-blind period, with similar percentages of subjects in the MPH ERCT and placebo groups reporting any AE. The most common TEAE reported during the double-blind period was URTI, 
reported by three subjects in each treatment group. No other $\mathrm{AE}$ was reported by more than one subject in the MPH ERCT group during the double-blind period. Pulse rate and blood pressure changes observed in this study were also consistent with previous reports on MPH safety and tolerability in children (Hammerness et al. 2009). No deaths, serious AEs, or suicidal ideation were observed during open-label or double-blind treatment with MPH ERCT.

This study had several notable strengths in its design, including the use of the laboratory school protocol to assess the efficacy of MPH ERCT under conditions that approximate an academic environment and activities appropriate for children 6-12 years of age. The study also used a dose-optimization procedure to ensure administration of clinically relevant doses of MPH ERCT. Finally, the study enrollment criteria allowed subjects who were treatment naive or had previously received treatment with stimulants, and children with inattentive, hyperactive/impulsive, or combined ADHD, approximating a real-world ADHD patient population.

Several limitations should also be noted. The exclusion of subjects with significant co-occurring psychiatric or medical illness may limit the generalizability of these findings to a wider patient population. Although comparable bioavailability has been demonstrated for $40 \mathrm{mg}$ MPH ERCT and $40 \mathrm{mg}$ IR MPH in a chewable tablet formulation given as two $20 \mathrm{mg}$ doses 6 hours apart (Abbas et al. 2016), no comparative data on the pharmacokinetics of the 20 and $60 \mathrm{mg}$ MPH ERCT doses are available. According to the study protocol, analyses for key secondary endpoints were adjusted for multiple comparisons; however, those for other secondary endpoints were not. In addition, no data were collected for analysis of patient socioeconomic status or intelligence, so effects of those factors could not be assessed in the current study. Likewise, no data were available to test the reliability between sites for the K-SADS. Furthermore, the study did not include an active comparator. Efficacy for MPH ERCT was demonstrated versus placebo, but direct comparison with other effective ADHD medications would require additional study. A direct comparison with another long-acting MPH formulation would be of particular interest, given that the estimated duration of effect for MPH ERCT differed for the 2 analyses performed. Finally, the study was not designed to assess the long-term efficacy or safety of treatment with MPH ERCT.

\section{Conclusions}

Efficacy of the first MPH ERCT was demonstrated in schoolaged children in a laboratory classroom setting. The average of all postdose SKAMP-Combined assessment scores was statistically significantly lower for subjects treated with MPH ERCT $20-60 \mathrm{mg}$ compared with placebo $(p<0.001)$, and significant improvement in impairment, based on SKAMP-Combined scores, was observed at 2 hours postdose. A treatment effect on PERMP total number of problems attempted and total number of problems correct was observed as early as 0.75 hours postdose and maintained through at least 8 hours postdose. The safety profile for MPH ERCT was similar to that of tablet, capsule, and suspension formulations of MPH ER; decreased appetite, headache, abdominal pain, mood swings, and irritability were the most common TEAEs during the 6-week, openlabel, dose-optimization period. URTI was the only AE reported by $>1$ subject in the MPH ERCT treatment group in the double-blind period. No serious AEs were observed during the study. The results of this phase 3 clinical trial indicate that MPH ERCT is a generally safe and well-tolerated treatment option for significantly reducing the symptoms of ADHD in children 6-12 years of age.

\section{Clinical Significance}

This is the first multicenter, phase 3 study of the efficacy and safety of a chewable tablet ER formulation of MPH. The results demonstrated a statistically significant improvement in behavior impairment in children with ADHD. Safety and tolerability findings from this study are consistent with those of other MPH ER formulations, including oral tablets and capsules, transdermal patch, and oral suspension. The potential availability of a safe and effective chewable MPH ER tablet would provide a new valuable formulation option, addressing an unmet need for patients with ADHD who dislike the other available formulations, cannot swallow tablets or capsules, or would prefer the convenience of a chewable tablet. A chewable long-acting MPH option may be a particularly suitable formulation for younger children with ADHD.

\section{Acknowledgments}

Medical writing support was provided by Kathleen M. Dorries, $\mathrm{PhD}$, at Peloton Advantage and was funded by Pfizer, Inc.

\section{Authors' Contributions}

Data analysis conducted by John Orazem.

\section{Disclosures}

Sharon Wigal receives or has received research support, acted as a consultant, been an advisory board member, and/or served on a speakers bureau for Addrenex Pharmaceuticals, Eli Lilly and Company, Forest Pharmaceuticals, McNeil Consumer \& Specialty Pharmaceuticals, NextWave Pharmaceuticals, NIMH, Noven, NuTec Pharma, Otsuka, Pfizer, Purdue, Quintiles, Rho, Rhodes Pharmaceuticals, Shionogi, Shire US, Sunovion, Taisho Pharmaceutical, and Tris Pharma. Ann Childress receives or has received research support, acted as a consultant, been an advisory board member, and/or served as a speaker for Shire Pharmaceuticals, Pearson, Pfizer, Noven, NextWave Pharmaceuticals, Lilly USA, Forest Research Institute, Otsuka, Sunovion, Ironshore, Rhodes, Theravance, Neurovance, Neos, Arbor, Tris Pharma, Purdue, Lundbeck, and Alcobra. Sally A. Berry is an employee of Tris Pharma and has served as a paid consultant for Pfizer in connection with the development of the manuscript. Heidi Belden was a paid consultant for Pfizer in connection with the development of this manuscript. Faith Walters, Phillip Chappell, Nancy A. Sherman, John Orazem, and Donna Palumbo are employees of Pfizer.

\section{References}

Abbas R, Palumbo D, Walters F, Belden H, Berry SA: Single-dose pharmacokinetics and relative bioavailability of a novel methylphenidate extended-release chewable tablet compared with immediaterelease methylphenidate chewable tablet. Clin Ther 38:1151-1157, 2016.

Adjei A, Teuscher NS, Kupper RJ, Chang WW, Greenhill L, Newcorn JH, Connor DF, Wigal S: Single-dose pharmacokinetics of methylphenidate extended-release multiple layer beads administered as intact capsule or sprinkles versus methylphenidate immediaterelease tablets $(\operatorname{Ritalin}((\mathrm{R})))$ in healthy adult volunteers. J Child Adolesc Psychopharmacol 24:570-578, 2014.

American Academy of Pediatrics: ADHD: Clinical Practice Guideline for the Diagnosis, Evaluation, and Treatment of Attention-Deficit/ Hyperactivity Disorder in Children and Adolescents. Pediatrics 128:1007-1022, 2011. 
American Psychiatric Association: Diagnostic and Statistical Manual of Mental Disorders, 5th ed. Washington, DC: American Psychiatric Association; 2013.

Aptensio XR [package insert]: Coventry (Rhode Island), Rhodes Pharmaceuticals, 2017.

Barkley RA: Major life activity and health outcomes associated with attention-deficit/hyperactivity disorder. J Clin Psychiatry 63(Suppl 12):10-15, 2002.

Birnbaum HG, Kessler RC, Lowe SW, Secnik K, Greenberg PE, Leong SA, Swensen AR: Costs of attention deficit-hyperactivity disorder (ADHD) in the US: Excess costs of persons with ADHD and their family members in 2000. Curr Med Res Opin 21:195-206, 2005.

Childress A, Sallee FR: The use of methylphenidate hydrochloride extended-release oral suspension for the treatment of ADHD. Expert Rev Neurother 13:979-988, 2013.

Childress AC, Brams M, Cutler AJ, Kollins SH, Northcutt J, Padilla A, Turnbow JM: The efficacy and safety of evekeo, racemic amphetamine sulfate, for treatment of attention-deficit/hyperactivity disorder symptoms: A multicenter, dose-optimized, double-blind, randomized, placebo-controlled crossover laboratory classroom study. J Child Adolesc Psychopharmacol 25:402-414, 2015.

Concerta [package insert]: Titusville (New Jersey), Janssen Pharmaceuticals, Inc., 2015.

Conners CK, Sitarenios G, Parker JD, Epstein JN: The revised Conners' Parent Rating Scale (CPRS-R): Factor structure, reliability, and criterion validity. J Abnorm Child Psychol 26:257-268, 1998.

Das D, Cherbuin N, Butterworth P, Anstey KJ, Easteal S: A populationbased study of attention deficit/hyperactivity disorder symptoms and associated impairment in middle-aged adults. PLoS One 7:e31500, 2012.

Daytrana [package insert]: Miami (Florida), Noven Therapeutics, LLC, 2015.

Feldman HM, Reiff MI: Clinical practice. Attention deficit-hyperactivity disorder in children and adolescents. N Engl J Med 370:838-846, 2014.

Fischer R, Schutz H, Grossmann M, Leis HJ, Ammer R: Bioequivalence of a methylphenidate hydrochloride extended-release preparation: Comparison of an intact capsule and an opened capsule sprinkled on applesauce. Int J Clin Pharmacol Ther 44:135-141, 2006.

Focalin XR [package insert]: East Hanover (New Jersey), Novartis Pharmaceuticals Corporation, 2015.

Gajria K, Lu M, Sikirica V, Greven P, Zhong Y, Qin P, Xie J: Adherence, persistence, and medication discontinuation in patients with attention-deficit/hyperactivity disorder-A systematic literature review. Neuropsychiatr Dis Treat 10:1543-1569, 2014.

Guy W: CGI Clinical Global Impressions. ECDEU Assessment Manual for Psychopharmacology Revised, 1976. US Department Health Education and Welfare. National Institute of Mental Health, 1976, pp. 217-222.

Hammerness P, Wilens T, Mick E, Spencer T, Doyle R, Mccreary M, Becker J, Biederman J: Cardiovascular effects of longer-term, highdose OROS methylphenidate in adolescents with attention deficit hyperactivity disorder. J Pediatr 155:84-89, 89, 2009.

International Council for Harmonisation: ICH harmonised tripartite guideline: Statistical principles for clinical trials E9. International Conference on Harmonisation, 1998. Available at www.ich.org/ products/guidelines/efficacy/efficacy-single/article/statistical-principlesfor-clinical-trials.html (last accessed March 30, 2017).

Jerome L, Segal A, Habinski L: What we know about ADHD and driving risk: A literature review, meta-analysis and critique. J Can Acad Child Adolesc Psychiatry 15:105-125, 2006.
Kaufman J, Birmaher B, Brent D, Rao U, Flynn C, Moreci P, Williamson D, Ryan N: Schedule for Affective Disorders and Schizophrenia for School-Age Children-Present and Lifetime Version (K-SADS-PL): Initial reliability and validity data. J Am Acad Child Adolesc Psychiatry 36:980-988, 1997.

Kimko H, Gibiansky E, Gibiansky L, Starr HL, Berwaerts J, Massarella J, Wiegand F: Population pharmacodynamic modeling of various extended-release formulations of methylphenidate in children with attention deficit hyperactivity disorder via meta-analysis. J Pharmacokinet Pharmacodyn 39:161-176, 2012.

Klassen AF, Miller A, Fine S: Health-related quality of life in children and adolescents who have a diagnosis of attention-deficit/ hyperactivity disorder. Pediatrics 114:e541-e547, 2004.

Lee L, Kepple J, Wang Y, Freestone S, Bakhtiar R, Wang Y, Hossain M: Bioavailability of modified-release methylphenidate: Influence of high-fat breakfast when administered intact and when capsule content sprinkled on applesauce. Biopharm Drug Dispos 24:233243, 2003.

Lee SS, Humphreys KL, Flory K, Liu R, Glass K: Prospective association of childhood attention-deficit/hyperactivity disorder (ADHD) and substance use and abuse/dependence: A meta-analytic review. Clin Psychol Rev 31:328-341, 2011.

Matza LS, Paramore C, Prasad M: A review of the economic burden of ADHD. Cost Eff Resour Alloc 3:5, 2005.

Mcgough JJ, Wigal SB, Abikoff H, Turnbow JM, Posner K, Moon E: A randomized, double-blind, placebo-controlled, laboratory classroom assessment of methylphenidate transdermal system in children with ADHD. J Atten Disord 9:476-485, 2006.

Meltzer EO, Welch MJ, Ostrom NK: Pill swallowing ability and training in children 6 to 11 years of age. Clin Pediatr (Phila) 45: 725-733, 2006.

Metadate CD [package insert]: Smyrna (Georgia), UCB, Inc., 2015.

Michele TM, Knorr B, Vadas EB, Reiss TF: Safety of chewable tablets for children. J Asthma 39:391-403, 2002.

Pelham WE, Gnagy EM, Burrows-Maclean L, Williams A, Fabiano GA, Morrisey SM, Chronis AM, Forehand GL, Nguyen CA, Hoffman MT, Lock TM, Fielbelkorn K, Coles EK, Panahon CJ, Steiner RL, Meichenbaum DL, Onyango AN, Morse GD: Once-aday Concerta methylphenidate versus three-times-daily methylphenidate in laboratory and natural settings. Pediatrics 107:E105, 2001.

Pentikis HS, Simmons RD, Benedict MF, Hatch SJ: Methylphenidate bioavailability in adults when an extended-release multiparticulate formulation is administered sprinkled on food or as an intact capsule. J Am Acad Child Adolesc Psychiatry 41:443-449, 2002.

Pliszka S: Practice parameter for the assessment and treatment of children and adolescents with attention-deficit/hyperactivity disorder. J Am Acad Child Adolesc Psychiatry 46:894-921, 2007.

Polaha J, Dalton WT, III, Lancaster BM: Parental report of medication acceptance among youth: Implications for everyday practice. South Med J 101:1106-1112, 2008.

Posner K, Brown GK, Stanley B, Brent DA, Yershova KV, Oquendo MA, Currier GW, Melvin GA, Greenhill L, Shen S, Mann JJ: The Columbia-Suicide Severity Rating Scale: Initial validity and internal consistency findings from three multisite studies with adolescents and adults. Am J Psychiatry 168:1266-1277, 2011.

Posner K, Oquendo MA, Gould M, Stanley B, Davies M: Columbia Classification Algorithm of Suicide Assessment (C-CASA): Classification of suicidal events in the FDA's pediatric suicidal risk analysis of antidepressants. Am J Psychiatry 164:1035-1043, 2007.

Quillivant XR [package insert]: New York, NextWave Pharmaceuticals, Inc.; a subsidiary of Pfizer, Inc., 2016.

Ritalin LA [package insert]: East Hanover (New Jersey), Novartis Pharmaceuticals Corporation, 2015. 
Robb AS, Findling RL, Childress AC, Berry SA, Belden HW, Wigal SB: Efficacy, safety, and tolerability of a novel methylphenidate extended-release oral suspension (MEROS) in ADHD. J Atten Disord 2014 [Epub ahead of print]; DOI: 10.1177/1087054714533191.

Sharma A, Couture J: A review of the pathophysiology, etiology, and treatment of attention-deficit hyperactivity disorder (ADHD). Ann Pharmacother 48:209-225, 2014.

Sugrue D, Bogner R, Ehret MJ: Methylphenidate and dexmethylphenidate formulations for children with attention-deficit/hyperactivity disorder. Am J Health Syst Pharm 71:1163-1170, 2014.

Swanson JM, Wigal SB, Wigal T, Sonuga-Barke E, Greenhill LL, Biederman J, Kollins S, Nguyen AS, Decory HH, Hirshe Dirksen SJ, Hatch SJ: A comparison of once-daily extended-release methylphenidate formulations in children with attention-deficit/hyperactivity disorder in the laboratory school (the COMACS Study). Pediatrics 113(3 Pt 1):e206-e216, 2004.

Visser SN, Danielson ML, Bitsko RH, Holbrook JR, Kogan MD, Ghandour RM, Perou R, Blumberg SJ: Trends in the parent-report of health care provider-diagnosed and medicated attention-deficit/ hyperactivity disorder: United States, 2003-2011. J Am Acad Child Adolesc Psychiatry 53:34-46, 2014.

Wigal SB, Childress AC, Belden HW, Berry SA: NWP06, an extended-release oral suspension of methylphenidate, improved attention-deficit/hyperactivity disorder symptoms compared with placebo in a laboratory classroom study. J Child Adolesc Psychopharmacol 23:3-10, 2013.
Wigal SB, Greenhill LL, Nordbrock E, Connor DF, Kollins SH, Adjei A, Childress A, Stehli A, Kupper RJ: A randomized placebocontrolled double-blind study evaluating the time course of response to methylphenidate hydrochloride extended-release capsules in children with attention-deficit/hyperactivity disorder. J Child Adolesc Psychopharmacol 24:562-569, 2014.

Wigal SB, Gupta S, Guinta D, Swanson JM: Reliability and validity of the SKAMP rating scale in a laboratory school setting. Psychopharmacol Bull 34:47-53, 1998.

Wigal SB, Kollins SH, Childress AC, Squires L: A 13-hour laboratory school study of lisdexamfetamine dimesylate in school-aged children with attention-deficit/hyperactivity disorder. Child Adolesc Psychiatry Ment Health 3:17, 2009.

Wigal SB, Wigal TL: The laboratory school protocol: Its origin, use, and new applications. J Atten Disord 10:92-111, 2006.

Wolraich ML, Mcguinn L, Doffing M: Treatment of attention deficit hyperactivity disorder in children and adolescents: Safety considerations. Drug Saf 30:17-26, 2007.

Address correspondence to: Sharon B. Wigal, PhD AVIDA, Inc.

1600 Dove Street, Suite 305 Newport Beach, CA 92660

E-mail: drsharon@avidainc.com 